\title{
Prospective analysis of myocardial strain through the evolution of Chagas disease in the hamster animal model
}

\author{
Fernando Fonseca França Ribeiro ${ }^{1}$ Henrique Turin Moreira ${ }^{1}$ • Antônio Carlos Leite de Barros-Filho ${ }^{1}$. \\ Denise M. Tanaka ${ }^{1}$. Camila G. Fabricio ${ }^{1}$ - Luciano F. L. Oliveira ${ }^{2}$. Cibele M. Prado ${ }^{3}$ - Marcus V. Simões ${ }^{1}$. \\ André Schmidt ${ }^{1}$. Benedito C. Maciel ${ }^{1}$. José A. Marin-Neto ${ }^{1} \cdot$ Minna Moreira Dias Romano $^{1}$ (i)
}

Received: 7 May 2021 / Accepted: 6 August 2021 / Published online: 18 September 2021

(c) The Author(s) 2021

\begin{abstract}
Speckle tracking echocardiography (STE) enables early diagnosis of myocardial damage by evaluating myocardial strain. We aimed to study sequential changes in structural and ventricular functional parameters during Chagas disease (CD) natural history in an animal model. 37 Syrian hamsters were inoculated intraperitoneally with Trypanosoma cruzi (Chagas) and 20 with saline (Control). Echocardiography was performed before the infection (baseline), at 1 month (acute phase), 4, 6, and 8 months (chronic phase) using Vevo 2100 (Fujifilm Inc.) ultrasound system. Left ventricular end-diastolic diameter, Left ventricular end-systolic diameter (LVESD), Left ventricular ejection fraction (LVEF), Global longitudinal (GLS), circumferential (GCS) and radial (GRS) strain were evaluated. Tricuspid annular plane systolic excursion (TAPSE) was used to assess right ventricular function. At 8 months, animals were euthanized and LV myocardial samples were analyzed for quantitation of inflammation and fibrosis. LVEF decreased over time in Chagas group and a difference from Control was detected at 6 months (p-value of groups\#time interaction $=0.005$ ). There was a pronounced decrease in GLS, GCS and TAPSE in Chagas group (p-value of groups\#time interaction $=0.003$ for GLS,$<0.001$ for GCS and $<0.009$ for TAPSE vs Control) since the first month. LVESD, LVEF and GLS were significantly correlated to the number of inflammatory cells $(r=0.41, p=0.046 ; r=-0.42, p=0.042 ; r=0.41, p=0.047)$ but not to fibrosis. In the Syrian hamster model of CD STE parameters (GLS and GCS) showed an early decrease. Changes in LVEF, LVESD, and GLS were correlated to myocardial inflammation but not to fibrosis.
\end{abstract}

Keywords Echocardiography $\cdot$ Speckle-tracking $\cdot$ Strain $\cdot$ Chagas cardiomyopathy $\cdot$ Hamsters $\cdot$ Animal experimental model

Minna Moreira Dias Romano

minna@fmrp.usp.br

Fernando Fonseca França Ribeiro

f.ffribeiro@gmail.com

Henrique Turin Moreira

hturin@yahoo.com.br

Antônio Carlos Leite de Barros-Filho

acbarrosfmrp@yahoo.com

Denise M. Tanaka

mayumi.denise@gmail.com

Camila G. Fabricio

camila_fabricio@hotmail.com

Luciano F. L. Oliveira

oliveiralfl@ hotmail.com

Cibele M. Prado

cibeleprado@usp.br
Marcus V. Simões

simoesmv78@gmail.com

André Schmidt

aschmidt@fmrp.usp.br

Benedito C. Maciel

bcmaciel@fmrp.usp.br

José A. Marin-Neto

marin_neto@yahoo.com

1 Cardiology Division, Internal Medicine Department, Cardiology Center of the Medical School of Ribeirão Preto, University of São Paulo Ribeirão Preto, Bandeirantes Avenue, 3900, Ribeirão Preto, São Paulo 14049-900, Brazil

2 Physical Therapy Department, Federal University of Minas Gerais, Belo Horizonte, Brazil

3 Animal Science and Food Engineering, University of São Paulo, USP, Pirassununga, São Paulo 13635-900, Brazil 


$\begin{array}{ll}\text { Abbreviations } & \\ \text { CCC } & \text { Chronic Chagas cardiomyopathy } \\ \text { STE } & \text { Speckle tracking echocardiography } \\ \text { LV } & \text { Left ventricle } \\ \text { CD } & \text { Chagas disease } \\ \text { PSLAX } & \text { Parasternal long-axis view } \\ \text { PSSAX } & \text { Parasternal short-axis view } \\ \text { LVEDD } & \text { Left ventricular end-diastolic diameter } \\ \text { LVESD } & \text { Left ventricular end-systolic diameter } \\ \text { LVEF } & \text { Left ventricular ejection fraction } \\ \text { LVEF_Teichholz } & \text { LVEF calculated by applying the } \\ & \text { method of Teichholz } \\ \text { LVEF_2D } & \text { LVEF calculated by bidimensional } \\ & \text { method of area-length } \\ \text { TAPSE } & \text { Tricuspid annular plane systolic } \\ & \text { excursion } \\ \text { GLS } & \text { Global longitudinal strain } \\ \text { GCS } & \text { Global circumferential strain } \\ \text { GRS } & \text { Global radial strain } \\ \text { ROI } & \text { Region of interest } \\ \text { RV } & \text { Right ventricle }\end{array}$

\section{Introduction}

Chagas disease (CD) remains one of the most prevalent infectious diseases in Latin America, where more than six million people are infected by the protozoan Trypanosoma cruzi $[1,2]$. The World Health Organization (WHO) recognizes CD as one of the thirteen most neglected tropical diseases in the world, with 25 million people at risk of infection $[3,4]$. Population interchange between endemic and non-endemic areas was responsible for the spread of the disease [5-7]. Consequently, CD has become an emerging public health issue also in non-endemic countries such as the United States and various European and Asian countries [8].

Chronic Chagas cardiomyopathy (CCC) is the most ominous manifestation of $\mathrm{CD}$ causing heart failure, conduction disturbances, ventricular arrhythmias, ventricular aneurysms, pulmonary and systemic thromboembolism, and sudden death $[9,10]$. The pathogenesis of CCC is pleomorphic and involves various mechanisms related to parasite persistence causing low grade but incessant inflammation and also myocardial injury mediated by the immune system. A particular aspect of the pathogenesis of CCC is delayed myocardial damage, which is related to a persistently low intensity but incessant parasitism and myocardial injury mediated by the immune system [11]. Most patients require specialized care, anticoagulation, pacemaker, cardioverterdefibrillator implantation and heart transplantation, with a high overall medical and social burden $[12,13]$.

It is estimated that roughly 30 to $40 \%$ of individuals infected by the T. cruzi will develop CCC only 20 to
30 years after the initial infection [14]. This life-long characteristic of the natural history of $\mathrm{CD}$, as also the absence of a prediction tool for who among infected people will develop CCC, is an unsolved challenge to clinical studies. In an attempt to overcome this problem, a model of $T$. cruzi infection in Syrian hamsters has been described to mimic all stages of $\mathrm{CD}$ with a more adequate timeline for research studies, aiming at the detection of early myocardial damage $[15,16]$.

Speckle tracking echocardiography (STE) has allowed the analysis of Left ventricle (LV) strain, a measurement of myocardial deformation, which has demonstrated higher accuracy for the detection of subclinical LV dysfunction in several clinical settings, such as cancer therapeutics-related cardiac dysfunction [17], idiopathic dilated cardiomyopathy [18-20], hypertrophic genetic cardiomyopathy [21], ischemic myocardial disease [22] and others [23]. The STE capability of diagnosing myocardial damage in the early stages of CD [24-26] and to predict evolution to CCC [27, $28]$ is still upon investigation.

The purpose of this study was to prospectively evaluate sequential changes in myocardial structure and function in an animal experimental model of CD using conventional and STE echocardiography compared to a control group. Specific objectives were (1) to examine the behavior of conventional echocardiographic systolic function parameters from both ventricles and LV myocardial deformation indices over lifelong evolution of animal experimental model of $\mathrm{CD}$, compared to controls and (2) to evaluate the correlation between conventional echocardiography parameters and LV myocardial deformation indices with histopathologic changes.

\section{Methods}

\section{Experimental animals}

Twelve-week-old female hamsters (Mesocricetus auratus) were kept in a climatically controlled environment, with free access to water and standard chow and under a 12-h light/dark cycle. Animals were subjected to all experimental procedures under anesthesia with Ketamine $(100 \mathrm{mg} / \mathrm{Kg})$ and Xylazine $(10 \mathrm{mg} / \mathrm{Kg})$ to avoid pain and stress. Experimental protocols were approved by the Institutional Animal Research Ethics Committee (Protocol No. 013/2015-1). Exclusion criteria were: inability to sedate with a recommended dose of anesthetics; clinical evidence of extracardiac pathologies; the presence of cardiac arrhythmias such as bigeminism, ventricular and supraventricular tachycardia, and evidence of cardiac abnormalities such as congenital defects. 


\section{Experimental protocol}

Animals were inoculated intraperitoneally with 35,000 trypomastigote forms of Trypanosoma cruzi (Y strain) and had chronic infection confirmed by plasma antibodies (Chagas group) or injected with an equal volume of saline solution (Control group). Echocardiography was performed before the inoculation (baseline) and at disease timepoints of acute (1 month) and chronic (4, 6, and 8 months after) phases. Animals were observed through eight months and survivors were subjected to euthanasia and heart collection for histological analysis. To demonstrate significant differences in echocardiographic parameters, specially LVEF, with adequate statistical power, taking into account a predicted mortality of 50\% in this experimental animal model [16], a minimum of 20 animals in each group would be necessary.

\section{Echocardiography}

Echocardiogram was recorded using Vevo 2100 (Fujifilm Inc.) ultrasound system provided with a $30-\mathrm{MHz}$ highfrequency transducer. Parasternal long-axis view (PSLAX) and short-axis view (PSSAX) were obtained. Left ventricular end-diastolic diameter (LVEDD), Left ventricular end-systolic diameter (LVESD), and Left ventricular ejection fraction (LVEF) were measured with M-mode at PSLAX. The LVEDD was measured in the maximum ventricular diastolic dimension and the LVESD at the maximum inward motion of the septum and posterior wall. LVEF was calculated by the method of Teichholz [29] (LVEF_Teichholz) and also by the bidimensional method of area-length (LVEF_2D). TAPSE was used to assess right ventricular function. All images were recorded by an echocardiographer experienced at imaging small animals at 5 to 10 min after sedation. All measurements represented the means of five consecutive cardiac cycles.

\section{Speckle tracking echocardiography}

Left ventricular Global longitudinal strain (GLS) was obtained at PSLAX. LV Global circumferential strain (GCS) and LV Global radial strain (GRS) were evaluated at PSSAX at the papillary level. Two-dimensional speckletracking analysis was applied to assess LV deformation using the validated Vevo® Strain Software (VisualSonics Inc, Toronto, Canada) [30]. End-diastole was automatically defined at the peak of the QRS complex. End-systolic time was defined as the point of the smallest dimension of the LV cavity and visually checked with the use of mitral leaflets position at M-mode background image. Manual LV endocardial border delineation was performed in the PSLAX to measure GLS or PSSAX to measure GRS and GCS. Thereafter, epicardial borders were automatically defined, creating a Region of interest (ROI). If necessary, adjustments in ROI width were made to include the entire LV myocardium region, based on a six-segment model [31] in each projection (Fig. 1).

Finally, myocardium tracking was automatically obtained. Also, a visual tracking quality control was applied and a new tracing was performed when at least one segment was poorly tracked. In the presence of a persistent poor tracking of at least one segment per projection, global deformation analysis was considered not feasible. LV deformation was measured as the peak systolic change in the myocardial length relative to the length at end-diastole and represented by a percentage of change (\%).

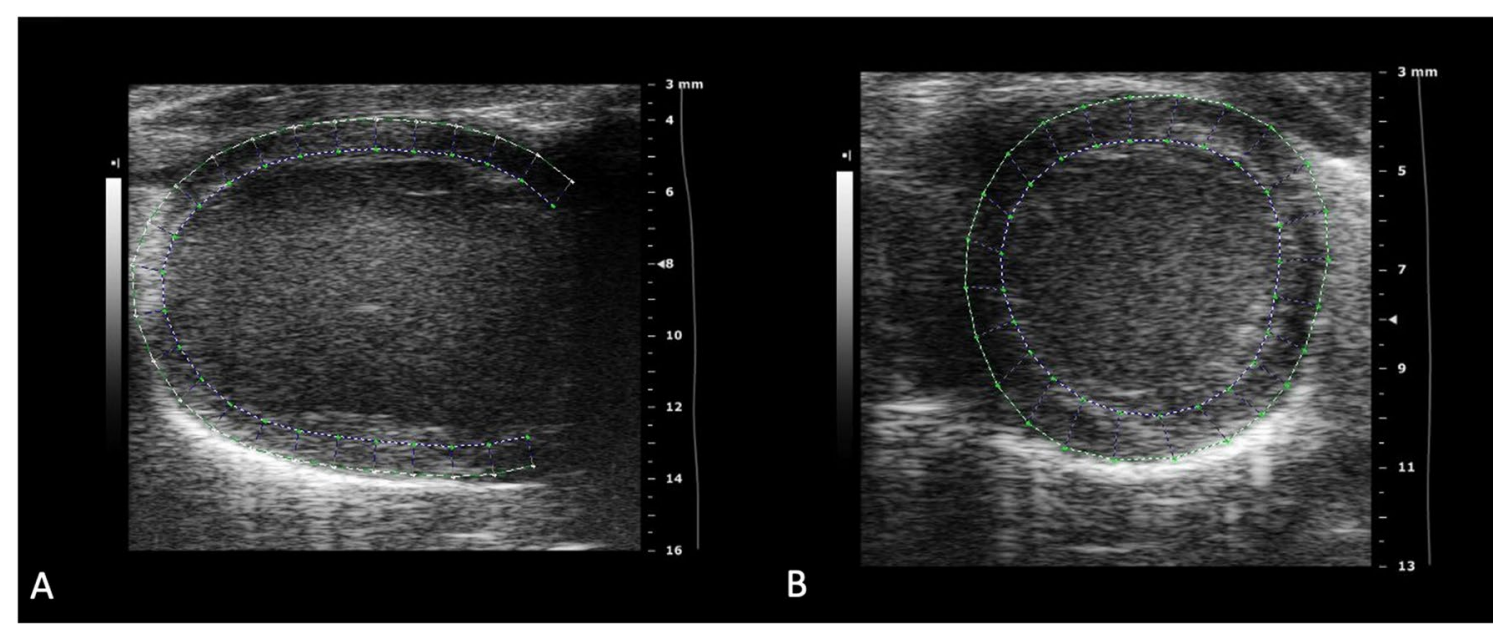

Fig. 1 Myocardial strain analysis in hamsters from Parasternal long-axis view (PSLAX) and Parasternal short-axis view (PSSAX). LV strain was measured across a Region of interest (ROI) in the parasternal long-axis view (A) and short-axis view (B) 


\section{Reproducibility}

Reproducibility analysis for echocardiographic parameters of LV functional assessment was performed in 20 animals randomly selected. For intraobserver reproducibility, a rereading was done at least 90 days after the first reading, blinded to the previous results. For interobserver analysis, the second independent reader was blinded to the analysis of the first reader. Inter- and intrareader reproducibility analyses were evaluated using an Intraclass correlation coefficient (ICC) to assess absolute agreement and a Coefficient of variation $(\mathrm{CV})$, determined by the standard deviation divided by the mean and expressed in percentage.

\section{Histopathological and morphometric analysis}

Animals were killed, the hearts were rapidly removed, rinsed in $0.9 \% \mathrm{NaCl}$ solution, and fixed in neutral buffered $10 \%$ formalin for histological study. For the histopathological study, the samples were dehydrated, clarified, embedded in paraffin, stained with hematoxylin and eosin and picrosirius red, and examined by light microscopy ( $n=10-13$ /group). The tissue sections stained with hematoxylin and eosin were used to evaluate the intensity of inflammation. The number of inflammatory cells was determined by counting the number of mononuclear rounded interstitial cells (to exclude the spindle-shaped fibroblastic cells) in the myocardium of the left ventricles in 10 microscope fields $(400 \times$ magnification) per animal. The slides stained with picrosirius red were used to evaluate fibrosis through collagen quantification. To estimate the volume fraction (\%) of fibrosis in picrosirius red-stained sections of the left ventricles, 10 microscope fields ( $400 \times$ magnifications) were measured per animal.

For both morphometric analyses, the Leica QWin software (Leica Imaging Systems Ltd., Cambridge, England) in conjunction with a Leica microscope, video camera, and an online computer was used. Measurements were made by a skilled observed blinded to the groups.

\section{Statistical analysis}

The normality of continuous data was assessed by histograms and the Shapiro-Wilk test. Continuous data are expressed as mean \pm standard deviation (SD) if normally distributed, or as median [interquartile range] if not normally distributed. Categorical data are presented as absolute values and percentages. Wilcoxon-Mann-Whitney tests were used to evaluate the differences between the two groups at baseline examination. Correlation of fibrosis and inflammation with echocardiographic parameters of LV and RV structural and functional assessment was verified by Pearson's correlation coefficient.

The analysis of variance for mixed models of repeated measures (mixed-ANOVA) was used to evaluate interaction (main effect) between the experimental groups (betweensubject effect) and time (within-subject effect). Statistical analyses were performed using Stata 14.0 (StataCorp, College Station, TX) and $\mathrm{P}<0.05$ was considered statistically significant.

\section{Results}

A total of 62 female Syrian hamsters was selected. Three animals were excluded from the basal evaluation, one of them because of thoracic deformity and two because of significant arrhythmias right after anesthesia. Two animals died during the first anesthesia. At baseline, 57 animals were subjected to echocardiography and there were no significant differences in characteristics between both groups, except for a higher weight of the Chagas group compared to controls $(143 \pm 12 \mathrm{~g}$ vs $130 \pm 15 \mathrm{~g} ; \mathrm{p}=0.004)$, Table 1 . The overall mortality of all animals was $49 \%$. The mortality of infected animals was $56.7 \%$, with $27 \%$ occurring up to the end of the first month after infection. Table 2 shows the number of live animals over time.

During the follow-up time, the sequential analysis showed that Chagas group had a significant increase in the LVESD and a decrease in LVEF_2D in comparison with Control

Table 1 Baseline population characteristics

\begin{tabular}{llll}
\hline & Chagas & Control & p-value \\
\hline $\mathrm{n}$ & 37 & 20 & \\
Age (days) & $89 \pm 1$ & $89 \pm 1$ & 0.551 \\
Weight (g) & $143 \pm 12$ & $130 \pm 15$ & $0.004^{*}$ \\
Heart rate (bpm) & $198 \pm 18$ & $204 \pm 18$ & 0.277 \\
LVEDD (mm) & $6.6 \pm 0.3$ & $6.6 \pm 0.3$ & 0.575 \\
LVESD (mm) & $4.4 \pm 0.4$ & $4.3 \pm 0.4$ & 0.503 \\
LVEF_Teichholz (\%) & $61 \pm 5$ & $64 \pm 5$ & 0.101 \\
LVEF_2D (\%) & $57 \pm 4$ & $57 \pm 4$ & 0.961 \\
TAPSE (mm) & $1.5 \pm 0.2$ & $1.5 \pm 0.2$ & 0.81 \\
GLS (\%) & $-14.2 \pm 3.4$ & $-15.2 \pm 2.7$ & 0.256 \\
GCS (\%) & $-20.4 \pm 2.8$ & $-20.0 \pm 2.2$ & 0.55 \\
GRS (\%) & $32.6 \pm 9.5$ & $33.7 \pm 10.4$ & 0.878 \\
\hline
\end{tabular}

LVEDD left ventricle end diastolic dimension, $L V E S D$ left ventricle end systolic dimension, $L V E F \_T e i c h h o l z$ left ventricular ejection fraction calculated by Teichholz method, $L V E F \_2 D$ two-dimensional left ventricular ejection fraction by area-length method, TAPSE tricuspid annular plane systolic excursion, $G L S$ global longitudinal strain, GCS global circumferential strain, GRS global radial strain $* \mathrm{p}<0.05$ 
Table 2 Number of live animals over time

\begin{tabular}{llllll}
\hline & Baseline & 1 Month & 4 Months & 6 Months & 8 Months \\
\hline Chagas (n) & 37 & 27 & 20 & 19 & 16 \\
Control (n) & 20 & 18 & 15 & 14 & 13 \\
Total (n) & 57 & 45 & 35 & 33 & 29 \\
\hline
\end{tabular}

group ( $\mathrm{p}$-value of the interaction groups \#time $=0.007$ for LVESD and of 0.003 for LVEF_2D) Fig. 2, Panels A and B.

Additionally, GLS and GCS of Chagas group showed a significant reduction over time in Chagas group compared to the Control group ( $\mathrm{p}$-value of the interaction

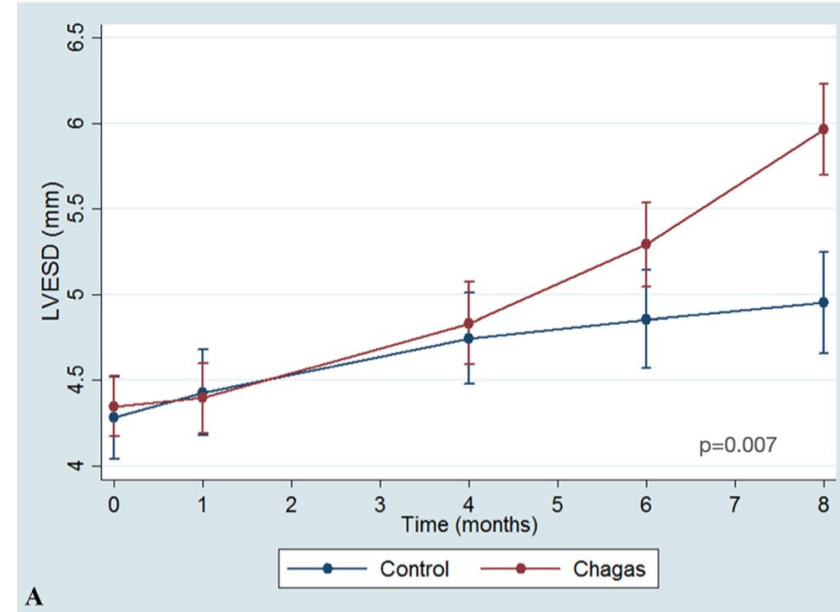

Fig. 2 Left ventricle end-systolic dimension (LVESD) and ejection fraction (LVEF) through time in Chagas and Control groups. Panel A Behavior of LVESD over time in Chagas and Control groups. Panel $\mathbf{B}$ LVEF over time in Chagas and Control groups. LVESD left ventricu-

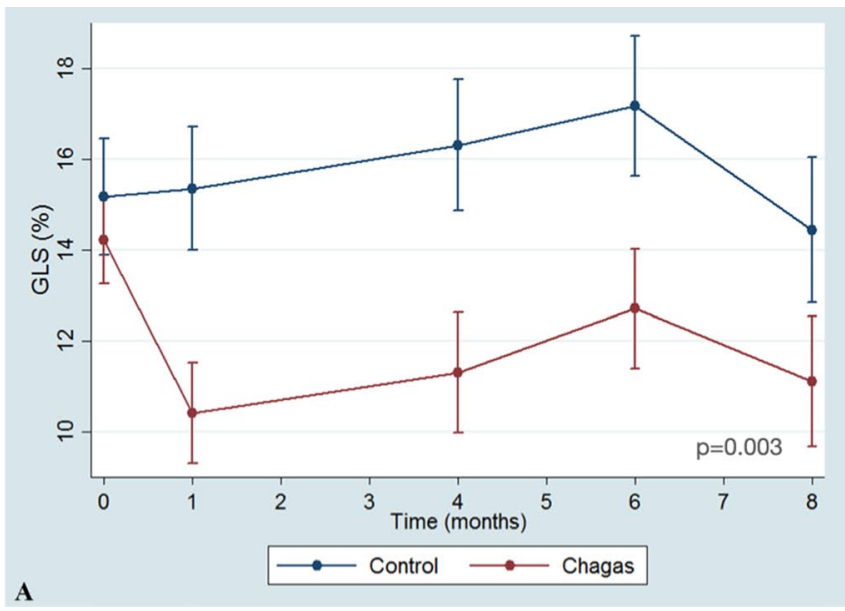

Fig. 3 Deformation echocardiographic parameters evolution over time in Chagas and Control groups. Panel A Evolution of GLS over time in Chagas and Control groups. Panel B Evolution of GCS over groups\#time $=0.003$ for GLS and $<0.001$ for GCS) - Fig. 3, Panels A and B. For both parameters of myocardial deformation, the difference between the two groups is verified from the first month of evaluation.

The evolution of both ventricles' structure and functional parameters is presented in Table 3 for Control group and in Table 4 for Chagas group. Values of LVEF_2D in Chagas group were $57 \pm 4 \%$ at baseline and reduced to $42 \pm 13 \%$ at 8 months. GLS in Chagas group was $-14.2 \pm 3.4 \%$ at baseline and reduced to $-10.4 \pm 3.0 \%$ from the first month.

The TAPSE index of the Chagas group also presented a significant reduction over time compared to the Control group (p-value of interaction groups\#time <0.009) - Fig. 4,

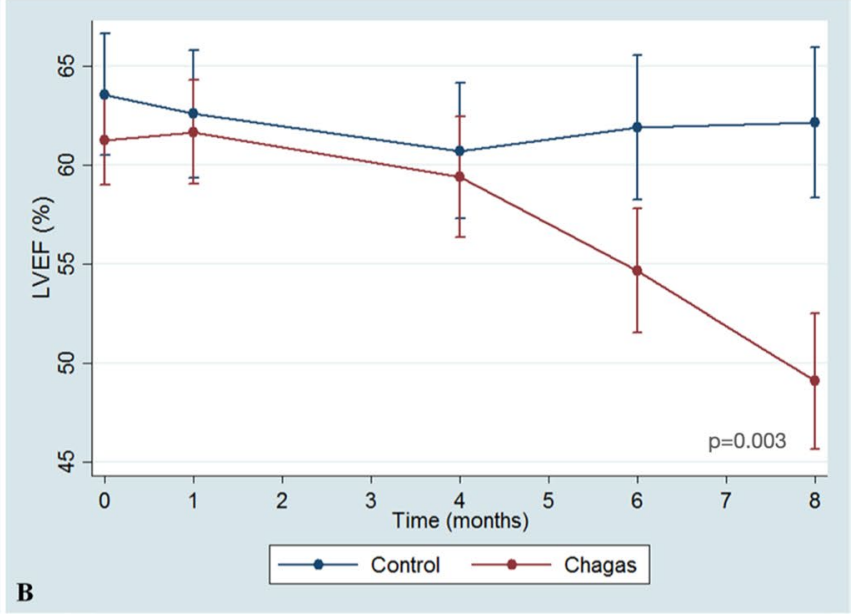

lar end-systolic diameter, $L V E F \_2 D$ two-dimensional derived left ventricular ejection fraction by the area-length method. Comparison between groups through time with mixed model ANOVA

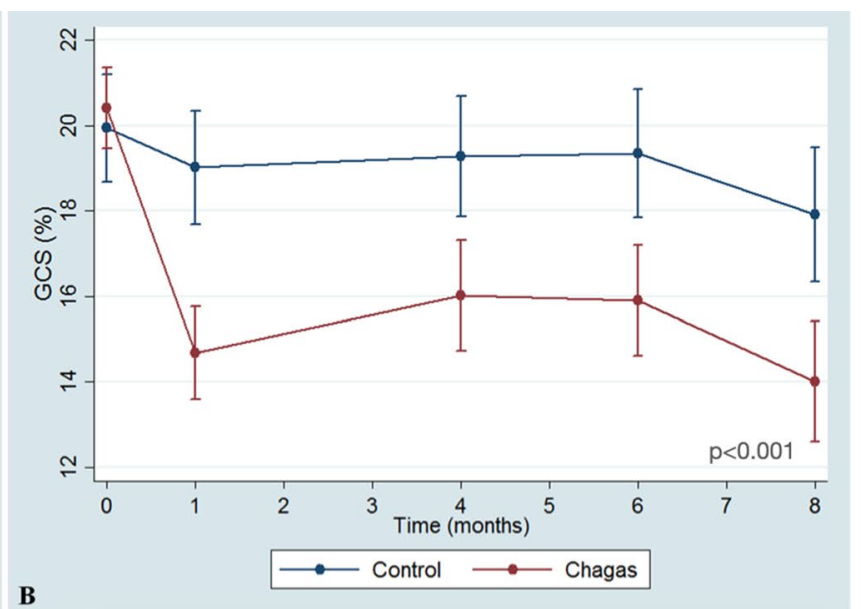

time in Chagas and Control groups. GLS global longitudinal strain, GCS: global circumferential strain. Comparison between groups through time with mixed model ANOVA 
Table 3 Sequential assessment of biventricular structure and function in the control group
Table 4 Sequential assessment of ventricular structure and function in the Chagas group

\begin{tabular}{lccccr}
\hline & \multicolumn{1}{l}{ Baseline } & \multicolumn{1}{l}{ 1 Month } & \multicolumn{1}{l}{ 4 Months } & \multicolumn{1}{l}{ 6 Months } & \multicolumn{1}{c}{8 Months } \\
\hline LVEDD (mm) & $6.6 \pm 0.3$ & $6.8 \pm 0.3$ & $7.2 \pm 0.3$ & $7.4 \pm 0.3$ & $7.6 \pm 0.5$ \\
LVESD (mm) & $4.3 \pm 0.4$ & $4.4 \pm 0.3$ & $4.7 \pm 0.3$ & $4.8 \pm 0.4$ & $4.9 \pm 0.5$ \\
LVEF_Teichholz (\%) & $63 \pm 5$ & $63 \pm 3$ & $61 \pm 4$ & $62 \pm 5$ & $62 \pm 5$ \\
LVEF_2D (\%) & $57 \pm 4$ & $57 \pm 3$ & $55 \pm 3$ & $57 \pm 6$ & $53 \pm 3$ \\
GLS (\%) & $-15.2 \pm 2.6$ & $-15.4 \pm 2.4$ & $-16.3 \pm 2.9$ & $-19.3 \pm 2.4$ & $-15.0 \pm 2.0$ \\
GCS (\%) & $-20.0 \pm 2.2$ & $-19.0 \pm 2.1$ & $-19.0 \pm 2.0$ & $-16.0 \pm 2.6$ & $-18.0 \pm 3.0$ \\
GRS (\%) & $34.0 \pm 10.0$ & $31.0 \pm 4.5$ & $29.0 \pm 6.0$ & $30.0 \pm 5.6$ & $29.0 \pm 9.3$ \\
TAPSE (mm) & $1.5 \pm 0.2$ & $1.7 \pm 0.2$ & $1.6 \pm 0.2$ & $1.7 \pm 0.1$ & $1.7 \pm 0.2$ \\
\hline
\end{tabular}

The analysis of variance (mixed-ANOVA) for mixed models of repeated measures was used to evaluate the differences between the two groups over time

LVEDD left ventricle end diastolic dimension, LVESD left ventricle end systolic dimension, LVEF_Teichholz left ventricular ejection fraction calculated by Teichholz method, $L V E F \_2 D$ two-dimensional left ventricular ejection fraction by area-length method, GLS global longitudinal strain, GCS global circumferential strain, GRS global radial strain, TAPSE tricuspid annular plane systolic excursion

\begin{tabular}{lcccrr}
\hline & \multicolumn{1}{l}{ Baseline } & \multicolumn{1}{c}{ 1 Month } & \multicolumn{1}{c}{ 4 Months } & \multicolumn{1}{c}{ 6 Months } & \multicolumn{1}{c}{8 Months } \\
\hline LVEDD (mm) & $6.6 \pm 0.3$ & $6.7 \pm 0.6$ & $7.2 \pm 0.4$ & $7.5 \pm 0.5$ & $8.0 \pm 0.6$ \\
LVESD (mm) & $4.4 \pm 0.4$ & $4.4 \pm 0.5$ & $4.8 \pm 0.5$ & $5.3 \pm 0.7$ & $6.0 \pm 1.2$ \\
LVEF_Teichholz (\%) & $61 \pm 5$ & $62 \pm 7$ & $59 \pm 6$ & $55 \pm 9$ & $49 \pm 14$ \\
LVEF_2D (\%) & $57 \pm 4$ & $55 \pm 6$ & $56 \pm 6$ & $48 \pm 8$ & $42 \pm 13$ \\
GLS (\%) & $-14.2 \pm 3.4$ & $-10.4 \pm 3.0$ & $-11.3 \pm 2.4$ & $-12.7 \pm 2.8$ & $-11.1 \pm 3.5$ \\
GCS (\%) & $-20.6 \pm 2.6$ & $-14.7 \pm 3.2$ & $-16.0 \pm 3.0$ & $-15.9 \pm 2.6$ & $-14.0 \pm 4.3$ \\
GRS (\%) & $32.6 \pm 9.5$ & $24.6 \pm 7.5$ & $23.8 \pm 6.5$ & $24.0 \pm 6.9$ & $22.7 \pm 8.8$ \\
TAPSE (mm) & $1.5 \pm 0.2$ & $1.3 \pm 0.2$ & $1.4 \pm 0.2$ & $1.4 \pm 0.2$ & $1.4 \pm 0.2$ \\
\hline
\end{tabular}

The analysis of variance (mixed-ANOVA) for mixed models of repeated measures was used to evaluate the differences between the two groups over time

LVEDD left ventricle end diastolic dimension, $L V E S D$ left ventricle end systolic dimension, LVEF_Teichholz left ventricular ejection fraction calculated by Teichholz method, $L V E F \_2 D$ two-dimensional left ventricular ejection fraction by area-length method, $G L S$ global longitudinal strain, $G C S$ global circumferential strain, GRS global radial strain, TAPSE tricuspid annular plane systolic excursion

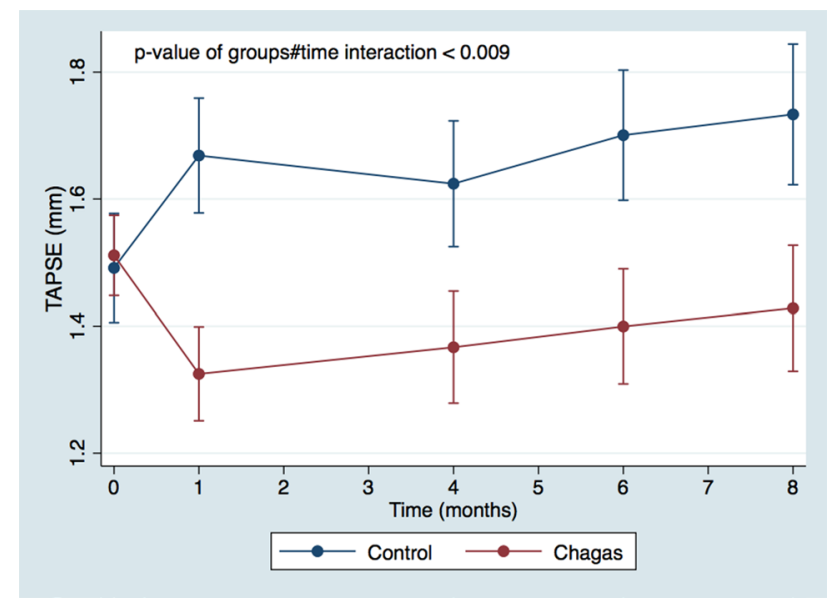

Fig. 4 Evolution of TAPSE over time in Chagas and Control groups. TAPSE tricuspid annular plane systolic excursion. Comparison between groups through time with mixed model ANOVA with the difference also observed since the first month post-infection.

\section{Histological analysis}

Histological analysis demonstrated diffuse myocarditis characterized by lymphomononuclear interstitial infiltrate in the Chagas group. There was an increase of $50 \%$ in the amount of mononuclear rounded interstitial cells $(30.30 \pm 10.9$ vs $20.5 \pm 9.46 ; p=0.045$ ) (Fig. 5).

The analysis of picrosirius red-stained sections revealed mild myocardial fibrosis manifested by an increased amount of pericellular collagen (endomysial matrix). The increase in the volume fraction of fibrosis was around 30\% (2.36 \pm 0.58 vs $1.82 \pm 0.29 ; \mathrm{p}=0.027$ ) (Fig. 6) in the Chagas group compared to the Control group. 
Fig. 5 Quantitative histological analysis of myocardial inflammation in Control group (A) and Chagas group (B). Graph representing the greater number of inflammatory cells in animals with Chagas disease compared to animals in the control group (C)

Fig.6 Quantitative histological analysis of interstitial myocardial fibrosis in Control group (A) and Chagas group (B). Graphic representing the highest percentage of interstitial fibrosis in animals with Chagas disease compared to animals in the control group (C)
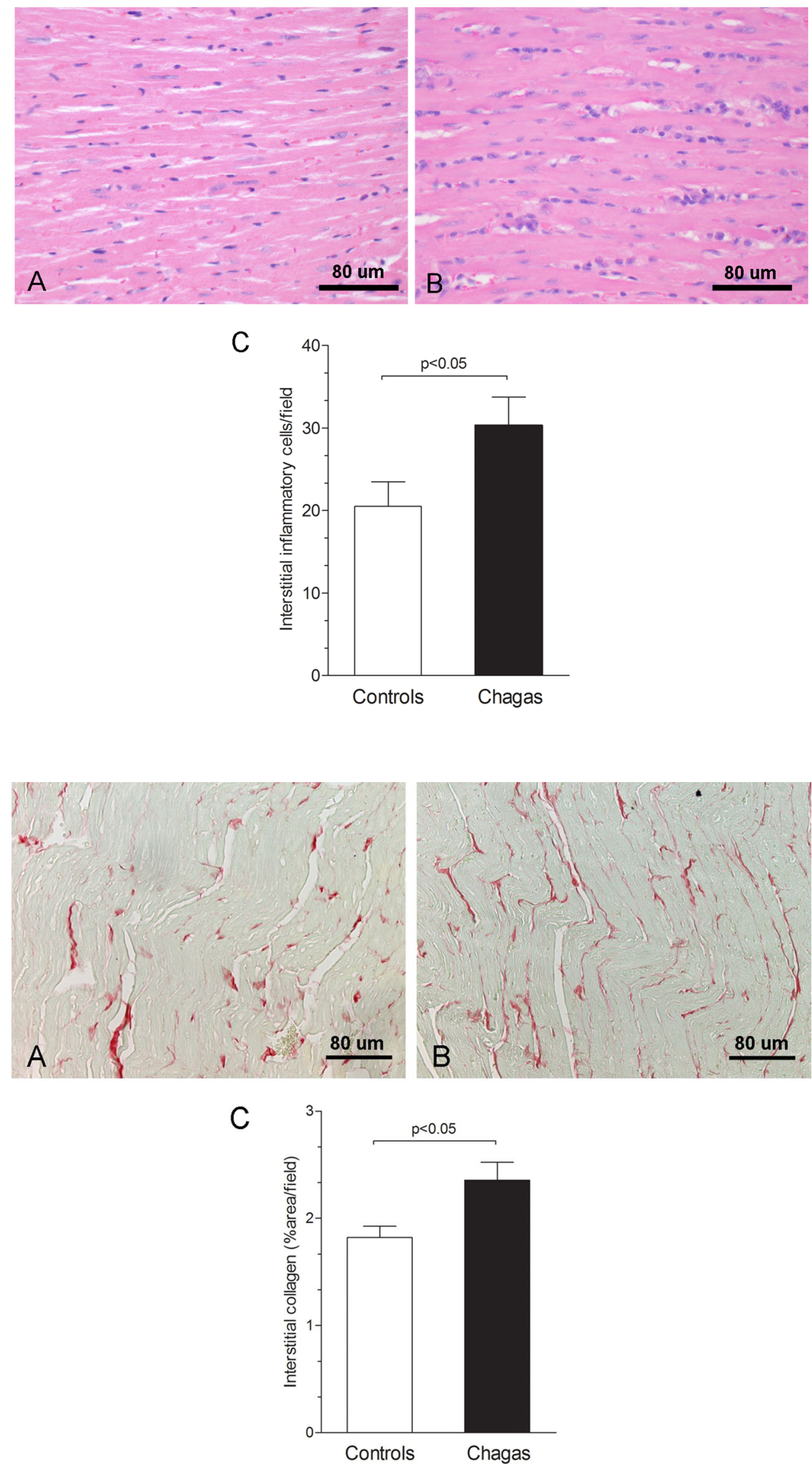
Table 5 Correlation of echocardiographic parameters with histological analysis

\begin{tabular}{lcllll}
\hline & \multicolumn{2}{l}{ Inflammation } & & \multicolumn{2}{l}{ Fibrosis } \\
\cline { 2 - 3 } \cline { 6 - 6 } \cline { 5 - 6 } & Pearson's r & p-value & & Pearson's r & p-value \\
\hline LVEDD & 0.28 & 0.19 & & 0.15 & 0.50 \\
LVESD & 0.41 & $0.046^{*}$ & & 0.18 & 0.42 \\
LVEF_Teichholz & -0.42 & $0.042^{*}$ & & -0.21 & 0.33 \\
LVEF_2D & -0.24 & 0.25 & & -0.13 & 0.54 \\
GLS & 0.41 & $0.047^{*}$ & -0.35 & 0.10 \\
GCS & 0.12 & 0.57 & & -0.33 & 0.13 \\
GRS & -0.22 & 0.30 & & -0.19 & 0.38 \\
TAPSE & -0.39 & 0.07 & & -0.36 & 0.10 \\
\hline
\end{tabular}

$L V E D D$ left ventricle end diastolic dimension, $L V E S D$ left ventricle end systolic dimension, $L V E F \_T e i c h h o l z$ left ventricular ejection fraction calculated by Teichholz method, $L V E F \_2 D$ two-dimensional left ventricular ejection fraction by area-length method, $G L S$ global longitudinal strain, $G C S$ global circumferential strain, $G R S$ global radial strain, TAPSE tricuspid annular plane systolic excursion

$* \mathrm{p}<0.05$

There was a significant moderate correlation between inflammation and GLS $(r=0.41, p=0.047)$, as also to LVEF_Teichholz $(\mathrm{r}=-0.42, \mathrm{p}=0.042)$ and LVESD $(\mathrm{r}=0.041, \mathrm{p}=0.046)$, Table 5. There was no significant correlation between systolic LV functional parameters and myocardial fibrosis.

\section{Reproducibility}

All echocardiographic parameters for LV functional assessment revealed good interobserver reproducibility, with the intraclass correlation coefficient ranging from 0.52 (for LVEF_2D) to 0.91 (for GLS). LVEF by Teichholz's method showed the lowest interobserver coefficient of variation (5.66\%), while GRS exhibited the highest (13.63\%).

Intraobserver reproducibility showed an intraclass correlation coefficient ranging from 0.49 (for LVEF_2D) to 0.92 (for GLS) and a coefficient of variation from $4.25 \%$ (for LVEF by Teichholz's method) to $10.85 \%$ (for GRS) (Table 6).

\section{Discussion}

The present study evaluated biventricular geometrical and functional ventricular changes in an experimental model of Chagas disease in Syrian hamsters over time in comparison with a Control group.

The findings revealed that parameters of longitudinal and circumferential myocardial deformation obtained by STE are significantly depressed early after the infection with $T$. cruzi. In contrast, LVESD and LVEF showed changes at an
Table 6 Reproducibility of echocardiographic parameters to assess LV systolic function

\begin{tabular}{llcllr}
\hline & \multicolumn{3}{l}{ Interobserver analysis } & \multicolumn{2}{l}{$\begin{array}{l}\text { Intraobserver } \\
\text { analysis }\end{array}$} \\
\cline { 2 - 3 } \cline { 5 - 6 } \cline { 5 - 6 } & ICC & CV (\%) & & ICC & CV (\%) \\
\hline LVEF (Teichholz) & 0.86 & 5.66 & & 0.91 & 4.25 \\
LVEF_2D & 0.52 & 7.24 & & 0.49 & 9.26 \\
LVEF (st) & 0.74 & 8.37 & & 0.90 & 5.53 \\
GLS & 0.91 & 11.10 & & 0.92 & 7.99 \\
GCS & 0.77 & 7.41 & & 0.85 & 7.10 \\
GRS & 0.67 & 13.63 & & 0.73 & 10.85 \\
\hline
\end{tabular}

$C V$ coefficient of variation, ICC intraclass correlation coefficient, LVEF (Teichholz) left ventricular ejection fraction by Teichholz method, $L V E F \_2 D$ two-dimensional left ventricular ejection fraction by area-length method, $L V E F(s t)$ left ventricular ejection fraction by speckle tracking method, GLS global longitudinal strain, GCS global circumferential strain, $G R S$ global radial strain

advanced time point of $\mathrm{CD}$, when mortality is high. Besides, the present investigation indicates an early involvement of the right ventricle in animals infected with $T$. cruzi, before the overt dysfunction of LV.

\section{Evolution of geometrical and biventricular functional parameters in the experimental animal model of $C D$ in hamsters}

Although the phases and forms of CD are difficult to be reproduced in rodent models of $T$. cruzi infection, in Syrian hamsters $C D$ can be replicated through all clinical stages including the acute phase, the latent period (indeterminate), the chronic cardiomyopathy, with myocardial dysfunction caused by multifocal and diffuse myocarditis and interstitial fibrosis. The first month after infection in hamsters presents the acute phase of the disease, with a high mortality rate. In our study, we observed $27 \%$ of mortality at this point and this finding is similar to that reported in a previous study, which showed a mortality rate of $33 \%$ in the first month [16]. After the acute phase, survivors evolve to an apparent quiescent period, with no clinical manifestations neither evidence of overt myocardial systolic dysfunction, similarly to the indeterminate form [32] of chronic CD in humans. Prior studies using the same animal experimental model did not find LV myocardial impairment with conventional echocardiography until 6 months after the initial infection [33]. In our study, LVEF and LVESD were reduced in infected animals compared to controls at 6 months of disease evolution, suggesting that the latent phase of CD in this experimental animal model may be shorter than previously reported $[15,16,34]$. It is also plausible to assume that those differences could be explained, at least in part, by the different 
echocardiographic techniques applied [15], which, in our study, used a higher spatial resolution ultrasound system.

Other studies reported on late LV dilatation in hamsters infected with $T$. cruzi [15]. In our study, LVEDD was not different in infected animals from controls until the end of experimentation time at 8 months. This finding was also observed in the investigation from Tanaka et al. and may suggest not only LVEDD is a very late marker of CCC but also it could not represent LV dysfunction [33]. Our study was the first to assess two-dimensional derived LVEF by the area-length method in T. cruzi infected Syrian hamsters. Previous studies had already shown the feasibility of twodimensional LVEF in mice and rat models of surgical myocardial overload, infarction, and LV dysfunction [35]. However, as CCC is typically a segmental disease [36], M-mode derived LVEF (Teichholz method) may not accurately estimate LV systolic dysfunction.

This was also the first study to prospectively evaluate RV function through the evolution of CD in Syrian hamsters. Our data showed RV is early compromised. These findings corroborate the results from studies in humans that have shown early changes of RV geometry and function even in the indeterminate form of CD [37-39]. The damage to the $\mathrm{RV}$ imposed by $\mathrm{CD}$ was previously demonstrated in studies of RV myocardial tissue samples [40] and in a murine model of disease [41] but only in our investigation a sequential evolution of RV dysfunction has been described over time.

\section{Speckle tracking echocardiography as a tool for early detection of myocardial dysfunction in an animal experimental model of CD}

This was the first study to use STE in Syrian hamsters infected with $T$. cruzi. Our results demonstrated that GLS and GCS were able to detect early myocardial damage in animals infected with the protozoan when compared with control animals at the same age. It is noteworthy that the change in these parameters occurred despite normal values of LVEF, as early as one month after infection, a moment recognized in this model as the acute phase of the $\mathrm{CD}$ when mortality rates are high.

There have been some studies with STE in patients with Chagas disease. However, the behavior of myocardial deformation through all stages of the disease in a sequential analysis had not been previously demonstrated. Although in CCC global LV deformation parameters and LVEF are reduced $[25,42]$, results are still conflicting about the early stages of the disease $[24,26,43]$. Few clinical studies evaluated LV myocardial deformation in patients with the indeterminate form of CD. One study showed that LV radial strain was reduced in 32 patients compared to controls, but statistical significance was achieved only at trend analysis [25]. Gomes et al. did not encounter STE differences between controls and patients at the early stages of the disease, except for a small group of seven patients who presented significant fibrosis diagnosed by cardiac magnetic resonance [24].

In contrast to the reduction of LV GLS and GCS observed in infected animals since the very early phase of the disease, GRS was not different between Chagas and control groups in our study. Radial strain interpretation has been challenging in several aspects. The wide variability of GRS indexes and also low reproducibility are recognized even in normal human hearts [44]. Also, when providing normal reference intervals of STE for the Syrian hamster model, GRS presented the lowest values of reproducibility [45]. Technically the lower reproducibility of GRS is comprehensible in any, human or small animal experimental images [46], based on the difficulty not to measure strain, but to track speckles in lateral lobes of bidimensional LV images. Measurements of radial strain are made from parasternal short-axis views, where segments in lateral lobes of the ultrasound beam do not have enough spatial resolution to provide adequate tracking [47], Additionally, radial deformation may be enhanced at the initial phases of myocardial damage, as a compensatory mechanism to the reduction of longitudinal and circumferential deformation [23, 48]. Thus, clinical use and interpretation of radial strain should be cautious. Lima et al., in concordance with our findings, also did not show differences in radial strain in Chagas patients at the early stages of the disease [49].

Measurements of myocardial deformation in this study strictly followed the latest published guidelines [48]. EKG signals were always recorded, end-systolic time was marked and strain peak was always measured as a systolic peak [47]. Definitions about what strain peak was measured were not always clarified in previous studies using STE in CD [25, 26]. Normal values of deformation parameters of $L V$ in Syrian hamsters were previously published by our research group [50]. In pathologies such as CCC, several LV conduction disturbances may be present and hence some LV segments can show post systolic peak deformations, which may not accurately represent patients' myocardial systolic function [23].

\section{Histological analysis}

Corroborating previous findings from our laboratory $[16,51]$ histologic data presented in this study show an increase of both, myocardial inflammatory cells and interstitial fibrosis in $T$. cruzi infected animals compared to controls. The low extent of myocardial fibrosis (4\%) corroborates this model as a representative of initial myocardial damage when studied until 8 months. Previous studies show fibrosis of $10 \%$ of the whole LV myocardial area when animals reach 10 months of infection [16]. Despite the lower incidence of fibrosis, GLS correlation to inflammatory cell count reaffirms this 
deformation parameter as an early marker of myocardial damage in Chagas disease's physiopathology, even before significant fibrosis. Other mechanisms which could be present in Chagas cellular damage as myocardial disarray [52, 53] were not explored in this study.

\section{Limitations}

Our findings should be interpreted in the context of the various limitations. First, the fact that only animals who had all echocardiographic analyses in pre-specified time points were included, according to the inherent characteristic of mixed-ANOVA analysis [54]. Although our experimental study design had the power to demonstrate significant differences between myocardial structural and functional echocardiographic parameters over time, there is a natural selection bias, because animals that spontaneously died before 8 months could not be included in the mixed-ANOVA analysis. Second, it is important to consider that anesthetic agents could influence clinical parameters such as heart rate, blood pressure, and, in the last instance, myocardial performance. Although isoflurane is considered by some authors as a more superficial anesthetic agent, it has significant effects on cardiac function evaluated by LVEF [55], ketamine has shown fewer effects on LVEF, and its results were already tested in hamsters [56]. There is a lack of data in the literature about the impact of anesthetic agents in deformation myocardial parameters, especially in this Syrian hamster experimental model. Nonetheless, there were no differences in cardiac HR between the two groups over time in our present investigation. Third, there was no LV diastolic analysis based on difficulties to acquire apical long-axis views of this animal model. Finally, there was no RV tissue analysis to correlate to functional echocardiographic parameters.

\section{Translational aspects and future perspectives}

Results of this investigation in the animal model of T. cruzi infection suggest STE as a potentially useful tool to study early sequential myocardial damage through $\mathrm{CD}$ evolution in humans. GRS and GLS can show myocardial impairment before the overt LV dysfunction shown by a reduction in LVEF. There is no robust evidence about how frequently echocardiographic examinations should be done in serological-positive patients without clinical signs of disease [57]. However, our experimental data suggest sequential STE evaluations could show early reductions in LV deformation parameters. STE could be useful in selecting patients that should be monitored closely and, eventually, select patients who could benefit from early treatment. Also, early detection of impairment in RV functional parameters may be a marker of disease progression from the indeterminate to the $\mathrm{CCC}$ form of CD.

Future studies delineated to investigate the prognostic value of STE in CD are warranted. Syrian hamster experimental animal model could provide insights to therapeutic effects of drugs in $T$. cruzi infection before LVEF reductions and testing reversibility of deformation parameters and myocardial function improvement.

\section{Conclusions}

In the Syrian hamster experimental model of $\mathrm{CD}$, the left ventricular STE parameters (GLS and GCS) showed an early decrease, and RV dysfunction was also detected even before overt LV dysfunction. Changes in LVEF, LVESD, and GLS were correlated to myocardial inflammation but not to fibrosis.

Author contributions Conception and design of the study, or acquisition of data, or analysis and interpretation of data: FFFR, HTM, ACLBF, DMT, CGF, LFLO, CMP, MMDR. Drafting the article or revising it critically for important intellectual content: FFFR, AS, BCM, MVS, JAMN, MMDR. Final approval of the version to be submitted: JAMN, MMDR.

Funding This work was supported by the "Fundação de Apoio a Pesquisa do Estado de São Paulo (FAPESP)-Brazil", [Grant number 2016/25403-9].

\section{Declarations}

Conflict of interest The authors declare no conflicts of interest.

Disclosure All authors have approved the final article.

Open Access This article is licensed under a Creative Commons Attribution 4.0 International License, which permits use, sharing, adaptation, distribution and reproduction in any medium or format, as long as you give appropriate credit to the original author(s) and the source, provide a link to the Creative Commons licence, and indicate if changes were made. The images or other third party material in this article are included in the article's Creative Commons licence, unless indicated otherwise in a credit line to the material. If material is not included in the article's Creative Commons licence and your intended use is not permitted by statutory regulation or exceeds the permitted use, you will need to obtain permission directly from the copyright holder. To view a copy of this licence, visit http://creativecommons.org/licenses/by/4.0/. 


\section{References}

1. Perez-Molina JA, Molina I (2018) Chagas disease. Lancet 391(10115):82-94. https://doi.org/10.1016/S0140-6736(17) 31612-4

2. Echeverria LE, Morillo CA (2019) American trypanosomiasis (Chagas disease). Infect Dis Clin North Am 33(1):119-134. https://doi.org/10.1016/j.idc.2018.10.015

3. Global Burden of Disease Study C (2015) Global, regional, and national incidence, prevalence, and years lived with disability for 301 acute and chronic diseases and injuries in 188 countries, 1990-2013: a systematic analysis for the global burden of disease study 2013. Lancet 386(9995):743-800. https://doi.org/10.1016/ S0140-6736(15)60692-4

4. Martins-Melo FR, Ramos AN Jr, Alencar CH, Heukelbach J (2016) Mortality from neglected tropical diseases in Brazil, 20002011. Bull World Health Organ 94(2):103-110. https://doi.org/10. 2471/BLT.15.152363

5. Bern C, Kjos S, Yabsley MJ, Montgomery SP (2011) Trypanosoma cruzi and Chagas' disease in the United States. Clin Microbiol Rev 24(4):655-681. https://doi.org/10.1128/CMR.00005-11

6. Requena-Mendez A, Aldasoro E, de Lazzari E, Sicuri E, Brown M, Moore DA et al (2015) Prevalence of Chagas disease in LatinAmerican migrants living in Europe: a systematic review and meta-analysis. PLoS Neg1 Trop Dis 9(2):e0003540. https://doi. org/10.1371/journal.pntd.0003540

7. Hotez PJ (2018) The rise of neglected tropical diseases in the "new Texas.” PLoS Negl Trop Dis 12(1):e0005581. https://doi.org/10. 1371/journal.pntd.0005581

8. Pinto Dias JC (2013) Human Chagas disease and migration in the context of globalization: some particular aspects. J Trop Med 2013:789758. https://doi.org/10.1155/2013/789758

9. Rassi A Jr, Marin JAN, Rassi A (2017) Chronic Chagas cardiomyopathy: a review of the main pathogenic mechanisms and the efficacy of aetiological treatment following the BENznidazole evaluation for interrupting trypanosomiasis (BENEFIT) trial. Mem Inst Oswaldo Cruz 112(3):224-235. https://doi.org/10.1590/ 0074-02760160334

10. Marin-Neto JA, Rassi Jr A, Simoes MV, Maciel BC, Schmidt A (2010) Chagas heart disease. In: Yusuf S, Cairns JA, Camm AJ, Fallen EL, Gersh BJ (Eds). Evidence-Based Cardiology. p. 18

11. Marin-Neto JA, Cunha-Neto E, Maciel BC, Simoes MV (2007) Pathogenesis of chronic Chagas heart disease. Circulation 115(9):1109-1123. https://doi.org/10.1161/CIRCULATIONAHA. 106.624296

12. Rassi A Jr, Rassi A, Marin-Neto JA (2009) Chagas heart disease: pathophysiologic mechanisms, prognostic factors and risk stratification. Mem Inst Oswaldo Cruz 104(Suppl 1):152-158. https:// doi.org/10.1590/s0074-02762009000900021

13. Lee BY, Bacon KM, Bottazzi ME, Hotez PJ (2013) Global economic burden of Chagas disease: a computational simulation model. Lancet Infect Dis 13(4):342-348. https://doi.org/10.1016/ S1473-3099(13)70002-1

14. Rassi A Jr, Rassi A, Marin-Neto JA (2010) Chagas disease. Lancet 375(9723):1388-1402. https://doi.org/10.1016/S0140-6736(10) 60061-X

15. Bilate AM, Salemi VM, Ramires FJ, de Brito T, Silva AM, Umezawa ES et al (2003) The Syrian hamster as a model for the dilated cardiomyopathy of Chagas' disease: a quantitative echocardiographical and histopathological analysis. Microbes Infect 5(12):1116-1124

16. de Oliveira LF, Romano MM, de Carvalho EE, Cabeza JM, Salgado HC, Fazan Junior R et al (2016) Histopathological correlates of global and segmental left ventricular systolic dysfunction in experimental chronic Chagas cardiomyopathy. J Am Heart Assoc. https://doi.org/10.1161/JAHA.115.002786

17. Kang Y, Xu X, Cheng L, Li L, Sun M, Chen H et al (2014) Twodimensional speckle tracking echocardiography combined with high-sensitive cardiac troponin $\mathrm{T}$ in early detection and prediction of cardiotoxicity during epirubicine-based chemotherapy. Eur J Heart Fail 16(3):300-308. https://doi.org/10.1002/ejhf.8

18. Meluzin J, Spinarova L, Hude P, Krejci J, Podrouzkova H, Pesl $M$ et al (2011) Estimation of left ventricular filling pressures by speckle tracking echocardiography in patients with idiopathic dilated cardiomyopathy. Eur J Echocardiogr 12(1):11-18. https:// doi.org/10.1093/ejechocard/jeq088

19. Matsumoto K, Tanaka H, Kaneko A, Ryo K, Fukuda Y, Tatsumi $\mathrm{K}$ et al (2012) Contractile reserve assessed by three-dimensional global circumferential strain as a predictor of cardiovascular events in patients with idiopathic dilated cardiomyopathy. J Am Soc Echocardiogr 25(12):1299-1308. https://doi.org/10.1016/j. echo.2012.09.018

20. de Gonzalo-Calvo D, Quezada M, Campuzano O, Perez-Serra A, Broncano J, Ayala R et al (2017) Familial dilated cardiomyopathy: a multidisciplinary entity, from basic screening to novel circulating biomarkers. Int J Cardiol 228:870-880. https://doi.org/10. 1016/j.ijcard.2016.11.045

21. Urbano-Moral JA, Rowin EJ, Maron MS, Crean A, Pandian NG (2014) Investigation of global and regional myocardial mechanics with 3-dimensional speckle tracking echocardiography and relations to hypertrophy and fibrosis in hypertrophic cardiomyopathy. Circ Cardiovasc Imaging 7(1):11-19. https://doi.org/10.1161/ CIRCIMAGING.113.000842

22. Asanuma T, Fukuta Y, Masuda K, Hioki A, Iwasaki M, Nakatani S (2012) Assessment of myocardial ischemic memory using speckle tracking echocardiography. JACC Cardiovasc Imaging 5(1):1-11. https://doi.org/10.1016/j.jcmg.2011.09.019

23. Collier P, Phelan D, Klein A (2017) A test in context: myocardial strain measured by speckle-tracking echocardiography. J Am Coll Cardiol 69(8):1043-1056. https://doi.org/10.1016/j.jacc.2016.12. 012

24. Gomes VA, Alves GF, Hadlich M, Azevedo CF, Pereira IM, Santos CR et al (2016) Analysis of regional left ventricular strain in patients with Chagas disease and normal left ventricular systolic function. J Am Soc Echocardiogr 29(7):679-688. https://doi.org/ 10.1016/j.echo.2016.03.007

25. Garcia-Alvarez A, Sitges M, Regueiro A, Poyatos S, Jesus Pinazo M, Posada E et al (2011) Myocardial deformation analysis in Chagas heart disease with the use of speckle tracking echocardiography. J Card Fail 17(12):1028-1034. https://doi.org/10.1016/j.cardf ail.2011.08.007

26. Barbosa MM, Costa Rocha MO, Vidigal DF, Bicalho Carneiro Rde C, Araujo RD, Palma MC et al (2014) Early detection of left ventricular contractility abnormalities by two-dimensional speckle tracking strain in Chagas' disease. Echocardiography 31(5):623-630

27. Santos Junior OR, da Costa Rocha MO, Rodrigues de Almeida F, Sales da Cunha PF, Souza SCS, Saad GP et al (2019) Speckle tracking echocardiographic deformation indices in Chagas and idiopathic dilated cardiomyopathy: incremental prognostic value of longitudinal strain. PLoS ONE 14(8):e0221028. https://doi.org/ 10.1371/journal.pone.0221028

28. Pazin-Filho A, Romano MM, Almeida-Filho OC, Furuta MS, Viviani LF, Schmidt A et al (2006) Minor segmental wall motion abnormalities detected in patients with Chagas' disease have adverse prognostic implications. Braz J Med Biol Res 39(4):483487. https://doi.org/10.1590/S0100-879X2006000400008

29. Teichholz LE, Kreulen T, Herman MV, Gorlin R (1976) Problems in echocardiographic volume determinations: 
echocardiographic-angiographic correlations in the presence of absence of asynergy. Am J Cardiol 37(1):7-11

30. Bauer M, Cheng S, Jain M, Ngoy S, Theodoropoulos C, Trujillo A et al (2011) Echocardiographic speckle-tracking based strain imaging for rapid cardiovascular phenotyping in mice. Circ Res 108(8):908-916. https://doi.org/10.1161/CIRCRESAHA.110. 239574

31. Morgan EE, Faulx MD, McElfresh TA, Kung TA, Zawaneh MS, Stanley WC et al (2004) Validation of echocardiographic methods for assessing left ventricular dysfunction in rats with myocardial infarction. Am J Physiol Heart Circ Physiol 287(5):H2049_ H2053. https://doi.org/10.1152/ajpheart.00393.2004

32. Marin-Neto JA, Almeida Filho OC, Pazin-Filho A, Maciel BC (2002) Indeterminate form of Chagas' disease. Proposal of new diagnostic criteria and perspectives for early treatment of cardiomyopathy. Arq Bras Cardiol 79(6):623-627

33. Tanaka DM, de Oliveira LFL, Marin-Neto JA, Romano MMD, de Carvalho EEV, de Barros Filho ACL et al (2019) Prolonged dipyridamole administration reduces myocardial perfusion defects in experimental chronic Chagas cardiomyopathy. J Nucl Cardiol 26(5):1569-1579. https://doi.org/10.1007/s12350-018-1198-7

34. Tanaka D, Carvalho E, Oliveira L, Romano M, Oliveira G, Barros-Filho A, et al. (Eds) (2016) Prolonged dipyridamole use is associated to amelioration of myocardial perfusion disturbance in experimental chronic Chagas cardiomiopathy. Eur J Heart Fail. WILEY-BLACKWELL 111 RIVER ST, HOBOKEN 070305774, NJ USA.

35. Ram R, Mickelsen DM, Theodoropoulos C, Blaxall BC (2011) New approaches in small animal echocardiography: imaging the sounds of silence. Am J Physiol Heart Circ Physiol 301(5):H1765-H1780. https://doi.org/10.1152/ajpheart.00559. 2011

36. Pazin A, Romano M, Almeida O, Furuta M, Viviani L, Schmidt A et al (2006) Minor segmental wall motion abnormalities detected in patients with Chagas' disease have adverse prognostic implications. Braz J Med Biol Res 39(4):483-487. https://doi.org/10. 1590/S0100-879X2006000400008

37. Marin-Neto JA, Marzullo P, Sousa AC, Marcassa C, Maciel BC, Iazigi $\mathrm{N}$ et al (1988) Radionuclide angiographic evidence for early predominant right ventricular involvement in patients with Chagas' disease. Can J Cardiol 4(5):231-236

38. Marin-Neto JA, Bromberg-Marin G, Pazin-Filho A, Simoes MV, Maciel BC (1998) Cardiac autonomic impairment and early myocardial damage involving the right ventricle are independent phenomena in Chagas' disease. Int J Cardiol 65(3):261-269

39. Moreira HT, Volpe GJ, Marin-Neto JA, Nwabuo CC, AmbaleVenkatesh B, Gali LG et al (2017) Right ventricular systolic dysfunction in chagas disease defined by speckle-tracking echocardiography: a comparative study with cardiac magnetic resonance imaging. J Am Soc Echocardiogr 30(5):493-502. https://doi.org/ 10.1016/j.echo.2017.01.010

40. Carrasco Guerra HA, Palacios-Pru E, Dagert de Scorza C, Molina C, Inglessis G, Mendoza RV (1987) Clinical, histochemical, and ultrastructural correlation in septal endomyocardial biopsies from chronic chagasic patients: detection of early myocardial damage. Am Heart J 113(3):716-724. https://doi.org/10.1016/00028703(87)90712-5

41. Jelicks LA, Shirani J, Wittner M, Chandra M, Weiss LM, Factor SM et al (1999) Application of cardiac gated magnetic resonance imaging in murine Chagas' disease. Am J Trop Med Hyg 61(2):207-214. https://doi.org/10.4269/ajtmh.1999.61.207

42. Acquatella $\mathrm{H}$ (2007) Echocardiography in Chagas heart disease. Circulation 115(9):1124-1131. https://doi.org/10.1161/CIRCU LATIONAHA.106.627323

43. Lima MS, Villarraga HR, Abduch MC, Lima MF, Cruz CB, Bittencourt MS et al (2016) Comprehensive left ventricular mechanics analysis by speckle tracking echocardiography in Chagas disease. Cardiovasc Ultrasound 14(1):20. https://doi.org/10. 1186/s12947-016-0062-7

44. Moreira HT, Nwabuo CC, Armstrong AC, Kishi S, Gjesdal O, Reis JP et al (2017) Reference ranges and regional patterns of left ventricular strain and strain rate using two-dimensional speckletracking echocardiography in a healthy middle-aged black and white population: the CARDIA study. J Am Soc Echocardiogr 30(7):647-58 e2. https://doi.org/10.1016/j.echo.2017.03.010

45. Barros Filho AL RF, Tanaka D, Moreira HT, Schmidt A, Simôes MV, Maciel BC, Marin-Neto JA, Romano MMD (2017) Feasibility, reference intervals and reproducibility for left ventricular ejection fraction and myocardial strain by speckle tracking echocardiography in adult Syrian Hamsters. J Am Soc Echocardiogr 30(6):1. https://doi.org/10.1016/j.echo.2017.04.007

46. Tee N, Gu Y, Murni SW (2015) Comparative myocardial deformation in three myocardial layers in mice by speckle tracking echocardiography. Biomed Res Int 2015:148501. https://doi.org/ 10.1155/2015/148501

47. Farsalinos KE, Daraban AM, Unlu S, Thomas JD, Badano LP, Voigt JU (2015) Head-to-head comparison of global longitudinal strain measurements among nine different vendors: the EACVI/ ASE inter-vendor comparison study. J Am Soc Echocardiogr 28(10):1171-1181. https://doi.org/10.1016/j.echo.2015.06.011

48. Voigt JU, Pedrizzetti G, Lysyansky P, Marwick TH, Houle H, Baumann R et al (2015) Definitions for a common standard for 2D speckle tracking echocardiography: consensus document of the EACVI/ASE/Industry task force to standardize deformation imaging. J Am Soc Echocardiogr 28(2):183-193. https://doi.org/ 10.1016/j.echo.2014.11.003

49. Lima MS, Voos MC, Mathias W Jr, Tsutsui JM (2017) Indeterminate form of Chagas disease: is left ventricular torsional mechanics a clue to subclinical myocardial abnormalities? J Echocardiogr 15(1):6-12. https://doi.org/10.1007/s12574-016-0305-5

50. Barros Filho ACL, Moreira HT, Dias BP, Ribeiro FFF, Tanaka DM, Schmidt A et al (2021) Feasibility and reference intervals assessed by conventional and speckle-tracking echocardiography in normal hamsters. Phys Rep 9(5):e14776. https://doi.org/10. $14814 /$ phy 2.14776

51. Lemos de Oliveira LF, Thackeray JT, Marin Neto JA, Dias Romano MM, Vieira de Carvalho EE, Mejia J et al (2018) Regional myocardial perfusion disturbance in experimental chronic Chagas cardiomyopathy. J Nucl Med 59(9):1430-1436. https://doi.org/10.2967/jnumed.117.205450

52. Baroldi G, Bigi R, Cortigiani L (2005) Ultrasound imaging versus morphopathology in cardiovascular diseases. Myocardial cell damage. Cardiovasc Ultrasound 3:32. https://doi.org/10.1186/ 1476-7120-3-32

53. Rossi MA (1991) The pattern of myocardial fibrosis in chronic Chagas' heart disease. Int J Cardiol 30(3):335-340

54. Sullivan LM (2008) Repeated measures. Circulation 117(9):12381243. https://doi.org/10.1161/CIRCULATIONAHA.107.654350

55. Pachon RE, Scharf BA, Vatner DE, Vatner SF (2015) Best anesthetics for assessing left ventricular systolic function by echocardiography in mice. Am J Physiol Heart Circ Physiol 308(12):H1525-H1529. https://doi.org/10.1152/ajpheart.00890. 2014

56. Tanaka DM, Romano MM, Carvalho EE, Oliveira LF, Souza HC, Maciel BC et al (2016) Effect of different anesthetic agents on left ventricular systolic function assessed by echocardiography in hamsters. Braz J Med Biol Res 49(10):e5294. https://doi.org/ 10.1590/1414-431X20165294

57. Nunes MCP, Badano LP, Marin-Neto JA, Edvardsen T, Fernandez-Golfin C, Bucciarelli-Ducci C et al (2018) Multimodality imaging evaluation of Chagas disease: an expert consensus of Brazilian cardiovascular imaging department (DIC) and the 
European association of cardiovascular imaging (EACVI). Eur Heart J Cardiovasc Imaging 19(4):459-460. https://doi.org/10. 1093/ehjci/jex 154
Publisher's Note Springer Nature remains neutral with regard to jurisdictional claims in published maps and institutional affiliations. 